\title{
LncZEB1-AS1 regulates hepatocellular carcinoma bone metastasis via regulation of the miR-302b-EGFR-PI3K- AKT axis
}

\author{
Zhen-jiang Ma ${ }^{1,2^{*}}$, Yao Wang ${ }^{3,4^{*}}$, Hui-fen $\mathrm{Li}^{5 *}$, Ming-Hua Liu ${ }^{3}$, Feng-rui Bi ${ }^{3}$, Long $\mathrm{Ma}^{3}$, Hui Ma ${ }^{\circledR}$ and \\ Hong-li Yan ${ }^{3 凶}$
}
1. Department of Orthopedics, the Third Affiliated Hospital of Second Military Medical University, Shanghai 201805, P.R. China.
2. Department of Orthopedics, Shanghai Ninth People's Hospital, Shanghai 200011, P.R. China.
3. Department of Laboratory Medicine, Changhai Hospital, Second Military Medical University, Shanghai, P.R. China.
4. Department of Laboratory Medicine, The Affiliated Wuxi Maternity and Child Health Care Hospital of Nanjing Medical University, Wuxi, Jiangsu 214000, China.
5. Department of Interventional, Eastern Hepatobiliary Surgery Hospital, Second Military Medical University, Shanghai, P.R. China.

*These authors contributed equally to this work.

$\square$ Corresponding authors: Hui Ma, Department of Orthopedics, the Third Affiliated Hospital of Second Military Medical University, Shanghai 201805, P.R. China; E-mail: huima@smmu.edu.cn; Hong-li Yan, Department of Laboratory Medicine, Changhai Hospital, Second Military Medical University, Shanghai, P.R. China; E-mail: hongliyan@smmu.edu.cn.

(c) The author(s). This is an open access article distributed under the terms of the Creative Commons Attribution License (https://creativecommons.org/licenses/by/4.0/). See http:/ /ivyspring.com/terms for full terms and conditions.

Received: 2020.03.13; Accepted: 2020.06.09; Published: 2020.06.28

\begin{abstract}
In patients with hepatocellular carcinoma $(\mathrm{HCC})$, disease progression and associated bone metastasis $(\mathrm{BM}) \mathrm{can}$ markedly reduce quality of life. While the long non-coding RNA (IncRNA) zinc finger E-box binding homeobox 1 antisense 1 (ZEBI-ASI) has been shown to function as a key regulator of oncogenic processes in HCC and other tumor types, whether it plays a role in controlling HCC BM remains to be established. In the current study, we detected the significant upregulation of IncZEBI-ASI in HCC tissues, and we found this expression to be associated with BM progression. When we knocked down this IncRNA in HCC cells, we found that this significantly reduced their migratory, invasive, and metastatic activity both in vitro and in vivo. At a mechanistic level, we found that IncZEBI-ASI was able to target miR-302b and to thereby increase PI3K-AKT pathway activation and EGFR expression, resulting in the enhanced expression of downstream matrix metalloproteinase genes in HCC cells. In summary, our results provide novel evidence that IncZEBI-ASI can promote HCC BM through a mechanism dependent upon the activation of PI3K-AKT signaling, thus highlighting a potentially novel therapeutic avenue for the treatment of such metastatic progression in HCC patients.
\end{abstract}

Key words: Hepatocellular carcinoma; Bone metastasis; LncZEB1-AS1; miR-302b; EGFR-PI3K-AKT axis

\section{Introduction}

Hepatocellular cancer (HCC) accounts for up to $90 \%$ of all liver cancer cases and is particularly prevalent in regions including sub-Saharan Africa and Eastern Asia [1, 2]. At present, HCC remains the sixth most common and third deadliest cancer type, with over 780,000 new cases and almost 750,000 deaths in 2012 alone [2]. Several major advances in recent years have helped to prolong HCC patient survival [3], but tumor metastasis is still associated with poor patient outcomes and limited long-term survival in those with [4]. Bone metastasis (BM) in particular is detected in roughly $38.5 \%$ of HCC patients exhibiting extrahepatic metastases [5, 6] and in $11.7 \%$ of HCC patients undergoing curative respective surgery [7]. $\mathrm{BM}$ is a serious HCC complication, as it is associated with the potential for bone fractures, severe pain, nerve compression [8], and a median survival of just 7.4 months [9]. Therefore, investigating the molecular mechanisms HCC BM is important will improve the diagnosis and treatment of this condition.

Long noncoding RNAs (lncRNAs) are RNAs which are $>200$ nucleotides long and which generally 
lack the potential to code for protein [10]. Dysregulated lncRNA expression profiles have been shown to be associated with a range of pathological processes including aberrant proliferation [11], angiogenesis [12], and tumor metastasis [13]. How lncRNAs regulate BM in HCC patients, however, is not well understood $[14,15]$. Most lncRNAs are found within cellular nuclei [16], wherein they regulate a number of biological activities at the transcriptional and posttranscriptional levels [17]. The zinc finger E-box binding homeobox 1 antisense 1 (ZEB1-AS1) lncRNA which is encoded in the 10p11.22 region contiguous with ZEB1 has been well-characterized as an oncogenic lncRNA [18]. ZEB1 is a key transcription factor associated with tumor cell metastasis, with lncZEB1-AS1 being an antisense transcript derived from the ZEB1 promoter [19]. Early studies found that lncZEB1-AS1 overexpression was evident in the context of esophageal squamous cell carcinoma [20], while more recent studies have confirmed that it is also dysregulated in colorectal cancer, gastric cancer, and HCC [18, 21, 22]. In gastric cancer patients, lncZEB1-AS1 overexpression has been found to be independently associated with reduced patient survival [23], whereas in colorectal cancer it is associated with enhanced tumor cell proliferation and migration through its regulation of the miR-101/ZEB1 axis [21]. Recent work by Li et al. [18] found that increased lncZEB1-AS1 expression was evident in HCC tumor tissue samples relative to adjacent normal tissues (ANTs) observed in HCC tissues, compared with the adjacent normal tissues, and they further demonstrated that this lncRNA was able to modulate HCC cellular proliferation, migration, invasion, and cell cycle progression. In a separate study, lncZEB1AS1 was suggested to regulate the progression of liver cancer owing to its ability to target miR-365a-3p [24]. Whether lncZEB1-AS1 similarly regulates HCC BM development or progression, however, remains to be determined.

In this study, we determined that lncZEB1-AS1 is able to promote HCC BM via the epigenetic suppression of miR-302b expression, resulting in enhanced EGFR/PI3K-AKT signaling. Together, our results suggest that this signaling axis may be a viable diagnostic and/or therapeutic target for clinicians seeking to prevent HCC BM development.

\section{Material and Methods}

\section{Patient samples}

We collected HCC and paired ANT samples from 90 patients with HCC undergoing respective surgery or tissue biopsy. These patients had not undergone any targeted therapeutic treatments prior to sample collection. Collected samples were snap-frozen and stored at $-80^{\circ} \mathrm{C}$. The research ethics committee of Eastern Hepatobiliary Surgery Hospital, Second Military Medical University approved this study, which was consistent with the Declaration of Helsinki. Patient characteristics and clinical findings are detailed in Table 1.

Table 1. Correlation of clinic-pathologic characteristics with LncZEBI-ASI level

\begin{tabular}{|c|c|c|c|}
\hline \multirow[t]{2}{*}{ Characteristics } & \multicolumn{2}{|l|}{ ZEB1-AS1 level } & \multirow[t]{2}{*}{$P$ value } \\
\hline & $\operatorname{High}(n=45)$ & Low $(n=45)$ & \\
\hline Age, y & & & 0.399 \\
\hline$\leq 50$ & 24 & 20 & \\
\hline$>50$ & 21 & 25 & \\
\hline Gender & & & 0.777 \\
\hline Male & 37 & 38 & \\
\hline Female & 8 & 7 & \\
\hline Liver cirrhosis & & & 0.378 \\
\hline Yes & 27 & 31 & \\
\hline No & 18 & 14 & \\
\hline $\mathrm{AFP}, \mathrm{ng} / \mathrm{mL}$ & & & 0.284 \\
\hline$\leq 20$ & 16 & 21 & \\
\hline$>20$ & 29 & 24 & \\
\hline Tumor differentiation & & & $0.011^{*}$ \\
\hline I-II & 5 & 15 & \\
\hline III-IV & 40 & 30 & \\
\hline Tumor size, cm & & & 0.266 \\
\hline$\leq 5$ & 26 & 29 & \\
\hline$>5$ & 19 & 16 & \\
\hline Tumor number & & & 0.517 \\
\hline Single & 36 & 38 & \\
\hline Multiple & 9 & 7 & \\
\hline Microvascular invasion & & & $0.030^{*}$ \\
\hline Yes & 29 & 38 & \\
\hline No & 16 & 7 & \\
\hline Pathological satellite & & & 0.694 \\
\hline Yes & 41 & 42 & \\
\hline No & 4 & 3 & \\
\hline Tumor encapsulation & & & $0.038^{*}$ \\
\hline Complete & 27 & 35 & \\
\hline None & 18 & 10 & \\
\hline Hepatitis B virus DNA & & & 0.527 \\
\hline$>1.0 \mathrm{e}+03 \mathrm{IU} / \mathrm{mL}$ & 23 & 20 & \\
\hline$<1.0 \mathrm{e}+03 \mathrm{IU} / \mathrm{mL}$ & 22 & 25 & \\
\hline BCLC stage & & & 0.581 \\
\hline $0-\mathrm{A}$ & 36 & 38 & \\
\hline B & 9 & 7 & \\
\hline TNM stage & & & 0.520 \\
\hline I & 25 & 28 & \\
\hline $\mathrm{II}+\mathrm{III}$ & 20 & 17 & \\
\hline
\end{tabular}

\section{Cell culture}

Human HCC (PLC, MHCC-97H, Hep3B, and Huh7) and control (NCM-460) cell lines were from the Chinese Academy of Sciences (Shanghai, China), and were cultured in DMEM (Gibco, NY, USA) containing 10\% FBS (Gibco) and penicillin/streptomycin (Sigma, $\mathrm{MO}, \mathrm{USA}$ ) at $37^{\circ} \mathrm{C}$ in a humidified $5 \% \mathrm{CO} 2$ incubator. PI3K activity was assessed as in a prior study [25]. 


\section{Transfection}

LncZEB1-AS1-specific siRNA constructs (si-ZEB1-AS1\#1, \#2) and non-targeting control constructs (si-NC) were from Guangzhou RIBOBIO Co., Ltd (Guangzhou, China), while agomir/ antagomir constructs specific for miR-302b and corresponding negative control constructs were from GenePharma Co., Ltd (Shanghai, China), as were the EGFR overexpression pcDNA3.1- EGFR (pc-EGFR) and empty control vectors. All cells were plated in 6well plates for $24 \mathrm{~h}$, followed by Lipofectamine 2000 (Invitrogen, USA)-mediated transfection.

\section{qRT-PCR}

Total RNA was isolated from cells with the TRIzol reagent (Invitrogen), and RNA quantity and quality were then analyzed via a NanoDrop device (NanoDrop Technologies, USA). The expression of miR-302b was assessed using a Mir- $X^{\mathrm{TM}}$ miRNA FirstStrand Synthesis Kit (TaKaRa Biotechnology, Dalian, China) and a Mir-X ${ }^{\mathrm{TM}}$ miRNA qRT-PCR TB Green ${ }^{\circledR}$ Kit (TaKaRa), while lncZEB1-AS1 and EGFR expression were analyzed using a PrimeScript ${ }^{\mathrm{TM}} \mathrm{RT}$ Reagent Kit (TaKaRa) and a SYBR-Green PCR Master Mix (TaKaRa). Relative mRNA and miRNA expression levels were quantified according to the $2-\Delta \Delta \mathrm{Cq}$ method, with actin and U6 being used as normalization controls, respectively. Primers were as follows: ZEB1-AS1-Forward: 5' - CCGTGGGCACTGC TGAAT-3', ZEB1-AS1-Reverse: 5'- CTGCTGGCAAG CGGAACT-3'; MMP2-Forward: 5' - TGACTTTCTTG GATCGGGTCG-3', MMP2-Reverse: 5'-AAGCACCA CATCAGATGACTG-3'; MMP7-Forward: 5'-GGTCA CCTACAGGATCGTATCATAT-3', MMP7-Reverse: 5'-CATCACTGCATTAGGATCAGAGGAA-3';

MMP9-Forward: 5'-TGTACCGCTATGGTTACACTC G-3', MMP9-Reverse: 5'-GGCAGGGACAGTTGCTTC T-3'; E-cadherin -Forward: 5'-TACACTGCCCAGGA GCCAGA-3', E-cadherin -Reverse: 5'-TGGCACCAGT GTCCGGATTA-3'; N-cadherin -Forward: 5'-TCAGG CGTCTGTAGAGGCTT-3', N-cadherin-Reverse: 5'-A TGCACATCCTTCGATAAGACTG-3'; VimentinForward: 5'-GACGCCATCAACACCGAGTT-3', Vimentin-Reverse: 5'-CTTTGTCGTTGGTTAGCTGG T-3'; miR-302b-Forward: 5'-ATCCAGTGCGTGTCGT G-3', miR-302b-Reverse: 5'-TGCTTAAGTGCTTCCAT GTT-3'; ACTIN-Forward: 5'-GGGAAATCGTGCGTG ACATTAAG-3', ACTIN-Reverse: 5'-GTGTTGGCGTA CAGGTCTTTG-3'; U6-Forward: 5'-CTCGCTTCGGC AGCACA-3', U6-Reverse: 5'-AACGCTTCACGAATT TGCGT-3'.

\section{CCK-8 assay}

After transfection, cells were incubated for $24 \mathrm{~h}$ prior to re-platting in 96-well plates $\left(2 \times 10^{3} /\right.$ well).
Cells were then incubated for the indicated periods of time, after which each well was treated using $10 \mu \mathrm{L}$ of CCK-8 solution (Shanghai Haling Biotechnology, Co., Ltd., Shanghai, China) at $37^{\circ} \mathrm{C}$ for $2 \mathrm{~h}$. Absorbance at $450 \mathrm{~nm}$ was then assessed via microplate reader (Bio-Rad, CA, USA).

\section{Transwell assays}

Cells were allowed to rest for $48 \mathrm{~h}$ following transfection, after which $5 \times 10^{4}$ cells were added to the upper chamber of an 8- $\mu \mathrm{m}$ pore size Transwell assay system (Corning, NY, USA) in $200 \mu \mathrm{L}$ of serum-free media. For invasion assays, the membrane of this chamber was coated using Matrigel (BD Biosciences, NJ, USA), while for migration assays it was not. A 600 $\mu \mathrm{L}$ aliquot of FBS-containing media (10\%) was added into the lower chamber for all assays, and cells were then incubated for 1 day. Non-migratory/invasive cells were gently removed with a swab, after which all remaining cells were methanol-fixed and stained with crystal violet. The cells in five random fields of view were then enumerated via microscopic analysis (Olympus Corporation, Tokyo, Japan).

\section{Bioinformatics analysis}

StarBase 3.0 (http://starbase.sysu.edu.cn/) was used to predict potential binding targets for lncZEB1-AS1.

\section{RNA immunoprecipitation (RIP)}

A Magna RIP RNA-Binding Protein Immunoprecipitation Kit (Millipore, MA, USA) was used based on provided directions in order to assess interactions between miR-302b and lncZEB1-AS1. Briefly, cellular lysates were mixed at $4{ }^{\circ} \mathrm{C}$ overnight with magnetic beads coated with a human AGO2 antibody or control IgG (Millipore). These beads were then collected, and qRT-PCR was used in order to analyze RNA enrichment in the precipitated fraction.

\section{Luciferase reporter assay}

We obtained lncZEB1-AS1 constructs containing wither wild-type (WT) or mutated (MUT) versions of the putative miR-302b binding site from GenePharma, and we then inserted these fragments into the pmirGLO Dual-luciferase Vector (Promega, WI, USA) in order to produce the WT- and MUT-ZEB1-AS1 reporter constructs. This same strategy was used for EGFR reporter plasmid preparation. Prior to transfection, cells were added to 24-well plates overnight and were allowed to grow until reaching $60-70 \%$ confluence. Cells were then co-transfected with reporter plasmids along with either agomir-302b or agomir-NC. Following an additional $48 \mathrm{~h}$ incubation, the Dual-Luciferase Reporter Assay System (Promega) was utilized according to provided 
instructions, with Renilla luciferase activity being used as a normalization control.

\section{Western blotting}

Total cellular protein was isolated with RIPA buffer (Beyotime Institute of Biotechnology), and a BCA assay kit (Beyotime Institute of Biotechnology) was then utilized to quantify protein levels in each sample. Equivalent protein amounts were separated by SDS-PAGE and transferred to PVDF membranes (Millipore). These blots were then blocked for $2 \mathrm{~h}$ with $5 \%$ non-fat milk, followed by $4{ }^{\circ} \mathrm{C}$ incubation overnight with appropriate primary antibodies. After being washed, blots were then incubated with an HRP-conjugated antibody (1:5,000; ab205718; Abcam), after which protein levels were visualized using an Immobilon Western Chemiluminescent HRP Substrate kit (Millipore). Primary antibodies were: rabbit polyclonal anti-EGFR (1:1000, ab5644, Abcam), anti-PTEN (1:1000, ab32199, Abcam), anti-Akt (\#9272, Cell Signaling Technology, USA), anti-phospho (Ser473)-Akt (1:2000, \#4060, Cell Signaling Technology), anti-MMP7 (1:1000, ab5706, Abcam), anti-MMP9 (1:1000, ab38898, Abcam), anti-ECadherin (1:1000, ab1416, Abcam), anti-N-Cadherin (1:1000, ab18203, Abcam), anti-Vimentin (1:1000, ab92547, Abcam).

\section{Pulmonary metastasis model}

The role of lncZEB1-AS1 in tumor cell metastasis was assessed using a model of pulmonary metastasis. Briefly, male 6-week-old mice were randomized into 4 groups ( $n=5$ each), and appropriate HCC cells were then intravenously injected via the tail vein. Metastases were evaluated in live mice at appropriate time points with an IVIS Lumina II system (Caliper Life Sciences, MA, USA) by injecting animals with 4 mg luciferin (Gold Biotech) in a $50 \mu \mathrm{L}$ saline volume and then imaging animals after 10 minutes. Following a 6 week period, all mice were sacrificed, and hematoxylin and eosin (H\&E) staining was used to visualize lung metastases directly. Animals were housed under standard conditions, and the guidelines of the Second Military Medical University Animal Care Facility and the National Institutes of Health were followed for all animal studies described herein.

\section{Statistical analysis}

Data are means \pm SD. Associations between lncZEB1-AS1 expression and patient clinicopathological findings were analyzed via chi-squared tests, while other data were compared via Student's t-tests or one-way ANOVAs with Tukey's post hoc test as appropriate. HCC patient survival was evaluated through a Kaplan-Meier analysis, with a log-rank test being used to gauge significance.
Correlations between gene expression patterns in HCC samples were assessed via Spearman's correlation analyses. $\mathrm{P}<0.05$ was the significance threshold.

\section{Results}

\section{HCC is associated with significant IncZEB 1-AS1 upregulation}

We began by evaluating levels of lncZEB1-AS1 expression in $90 \mathrm{HCC}$ patient tumor tissues and paired ANTs via qRT-PCR, revealing significant upregulation of this lncRNA specifically within tumor samples (Figure 1A), with this upregulation being significant in 72/90 (80.0\%) samples (Figure 1B). We also determined that lncZEB1-AS1 expression levels were significantly higher in HCC patients with extrahepatic metastases (EHMH; $n=32)$ relative to those with metastasis-free HCC (MFH; $n=58$ ) (Figure 1C). Consistent with these findings, we determined that the MHCC-97H and Huh-7 HCC cell lines expressed higher levels of lncZEB1-AS1 relative to control liver cell lines, and were more invasive and metastatic [26] (Figure 1D). These data confirmed that lncZEB1-AS1 upregulation was thus closely linked to HCC metastasis.

\section{Upregulation of IncZEB 1-AS1 is closely linked to $B M$ and poorer outcomes in HCC patients}

We next explored the relevance of lncZEB1-AS1 to HCC patient metastatic progression and survival outcomes. To that end, we separated the abovementioned 90 HCC patients into lncZEB1-AS1high and -low groups according to median levels of this lncRNA. This analysis revealed that high lncZEB1-AS1 expression was significantly associated with microvascular invasion, poor differentiation, and a lack of encapsulation (Table 1). In a univariate analysis, we found tumor encapsulation, vascular invasion, BCLC stage, and lncZEB1-AS1 levels were all associated with BM in this patient cohort (Table 2). A subsequent multivariate analysis indicated that vascular invasion, BCLC stage, and lncZEB1-AS1 expression were all independently associated with the risk of BM in HCC patients (Table 2). We then confirmed this relationship using an independent TCGA dataset of 362 HCC patients that was analyzed with the GEPIA program, confirming that higher lncZEB1-AS1 expression was significantly linked to poorer overall survival $(\mathrm{n}=362$, hazard ratio $(\mathrm{HR})=$ 1.7, $\mathrm{P}(\mathrm{HR})=0.0026$, log-rank $\mathrm{P}=0.0024)$ and disease-free survival $(\mathrm{n}=362, \mathrm{HR}=1.6, \mathrm{P}(\mathrm{HR})=$ 0.0019 , log-rank $\mathrm{P}=0.0018$ ) (Figure $1 \mathrm{E}$ and $\mathrm{F}$ ). These results thus suggested that lncZEB1-AS1 may be an important regulator of BM in HCC patients. 
A

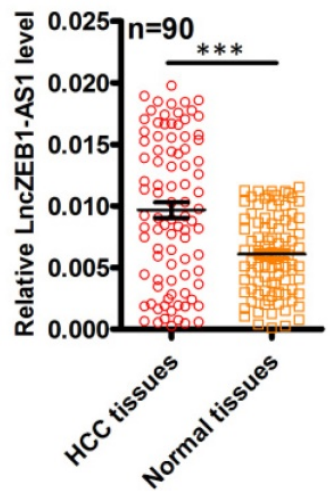

$\mathrm{D}$

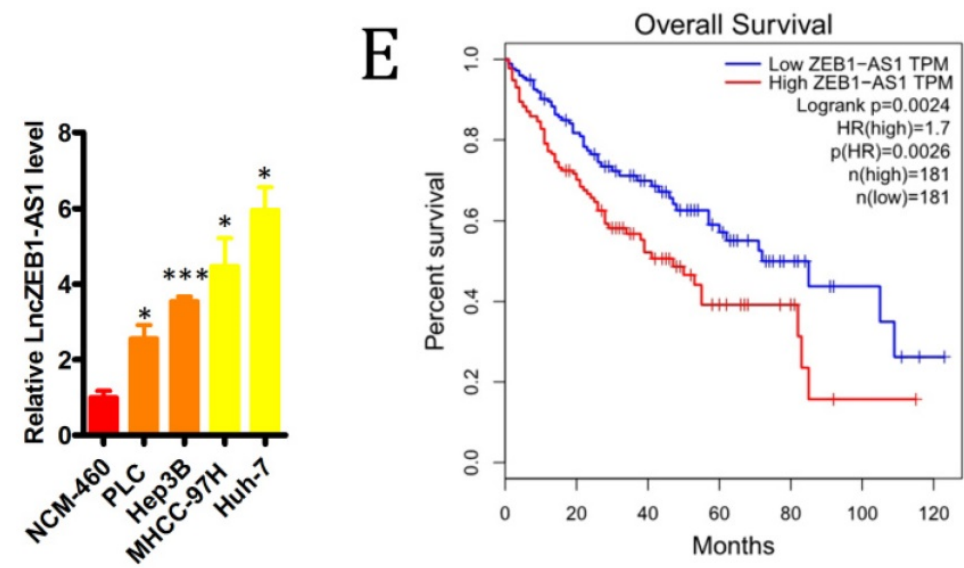

B

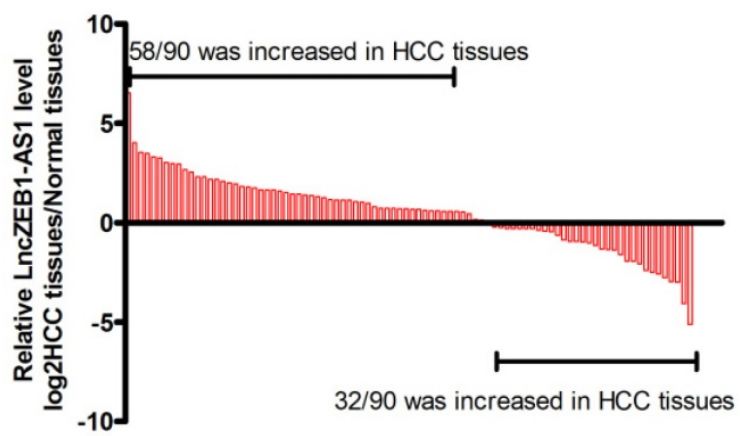

C

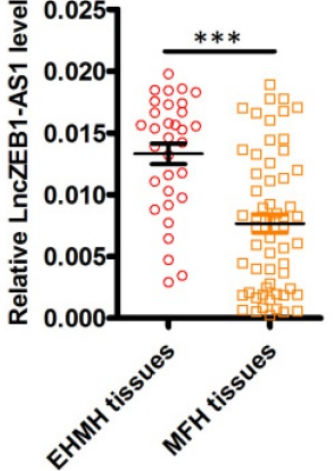

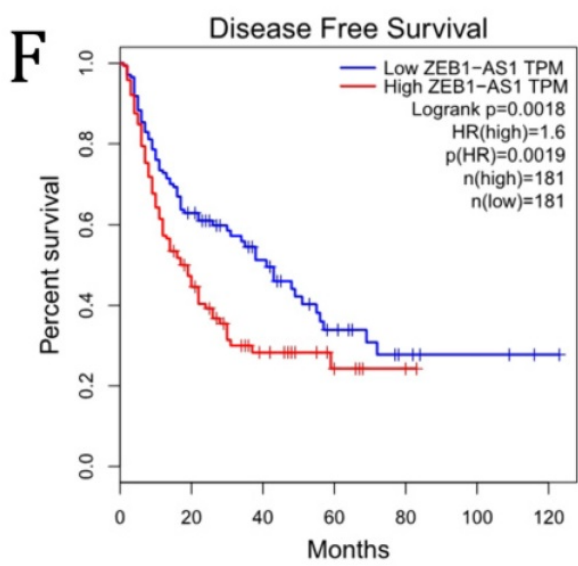

Figure 1. The upregulation of IncZEB 1 -ASI is associated with HCC BM and poor patient prognosis. (A and B) LncZEBI-AS1 expression was assessed via qRT-PCR in 90 pairs of HCC tumor samples and ANTs. C, LncZEB1-AS1 expression in patients with MFH ( $n=58)$ and EHMH ( $n=32$ ) tissues. (D) LncZEB1-AS1 levels were compared via qRT-PCR in control or HCC (MHCC-97H, Huh-7, PLC, Hep3B) human cell lines. Data for A-D are means \pm SEM and were compared via Student's t-tests. (E and F) Correlations between IncZEBI-ASI expression and HCC patient overall and disease-free survival were compared via a two-sided log-rank test using data from the GEPIA database. ${ }^{*} \mathrm{p}<0.05 ; *_{\mathrm{p}}<0.01 ; * * * \mathrm{*}<0.001$.

Table 2. Univariate and multivariate analyses of factors associated with bone metastasis in $90 \mathrm{HCC}$ patients

\begin{tabular}{|c|c|c|c|c|c|c|}
\hline \multirow[t]{2}{*}{ Variable Bone metastasis } & \multicolumn{3}{|c|}{ Univariate analyses } & \multicolumn{3}{|c|}{ Multivariate analyses } \\
\hline & $\mathrm{HR}$ & $95 \% \mathrm{CI}$ & $\mathrm{P}$ & $\mathrm{HR}$ & $95 \% \mathrm{CI}$ & $\mathrm{P}$ \\
\hline Age $>50$ versus $\leq 50$ years & 1.867 & $0.720-2.304$ & 0.107 & - & - & - \\
\hline Gender male versus female & 0.702 & $0.629-1.866$ & 0.968 & - & - & - \\
\hline Liver cirrhosis Yes versus No & 1.771 & $0.733-2.625$ & 0.551 & - & - & - \\
\hline $\mathrm{AFP}, \mathrm{ng} / \mathrm{mL}>20$ versus $\leq 20$ & 1.463 & $0.419-2.829$ & 0.114 & - & - & - \\
\hline Tumor differentiation III-IV versus I-II & 1.892 & $0.563-3.317$ & 0.631 & - & - & - \\
\hline Tumor size, $\mathrm{cm}>5$ versus $\leq 5$ & 0.684 & $0.458-1.534$ & 0.345 & - & - & - \\
\hline Tumor number multiple versus single & 1.398 & $0.582-1.964$ & 0.406 & - & - & - \\
\hline Vascular invasion Yes versus No & 2.492 & $1.312-3.649$ & 0.031 & 2.112 & $1.314-3.162$ & 0.034 \\
\hline Pathological satellite Yes versus No & 1.323 & $0.739-3.962$ & 0.629 & - & - & - \\
\hline Tumor encapsulation complete versus none & 2.625 & $1.739-4.404$ & 0.022 & 1.892 & $0.537-2.461$ & 0.234 \\
\hline Hepatitis B virus DNA, $\mathrm{IU} / \mathrm{mL}>1.0 \mathrm{e}+03$ versus $<1.0 \mathrm{e}+03$ & 1.367 & $0.791-2.169$ & 0.932 & - & - & - \\
\hline BCLC stage $B$ versus $0-A$ & 1.702 & $1.371-3.194$ & 0.019 & 1.526 & $1.712-2.622$ & 0.045 \\
\hline TNM stage II + III versus I & 1.524 & $0.394-2.417$ & 0.338 & - & - & - \\
\hline LncZEB1-AS1 high versus low & 2.767 & $1.460-2.061$ & 0.009 & 2.278 & $1.240-3.507$ & 0.015 \\
\hline
\end{tabular}

\section{LncZEB 1-AS1 knockdown impairs the growth and metastasis of HCC cells}

In order to explore the functional roles of lncZEB1-AS1 in HCC, we next modulated the expression of this lncRNA in vitro in HCC cells. We utilized specific shRNA constructs to knock down lncZEB1-AS1 in Huh7 and MHCC-97H cells, confirming successful knockdown via qRT-PCR (Figure 2A). Knockdown of this lncRNA was shown to markedly impair the proliferation of these HCC cells in a CCK-8 assay (Figure 2B), and to suppress tumor cell invasion and migration in a Transwell assay system (Figure 2C and D). To explore the in vivo relevance of these findings, we next utilized a murine model of pulmonary metastasis in which mice were 
injected with specific HCC cell lines. Metastatic nodules in the lungs of these mice were imaged every 2-3 weeks, revealing that these metastases grew much more slowly in animals injected with HCC cells in which lncZEB1-AS1 had been knocked down (Figure $2 \mathrm{E}$ and $\mathrm{F}$ ). Following a 6-week period, these animals were sacrificed, and H\&E staining of collected lungs confirmed that the knockdown of this lncRNA in HCC cells was associated with significantly reduced micro-metastasis formation in these animals (Figure $2 \mathrm{G}$ and $\mathrm{H})$. Together, these results thus provide strong evidence that lncZEB1-AS1 facilitates the development and/or progression of HCC.

\section{LncZEB 1-AS1 promotes PI3K-AKT signaling in order to induce MMP-2, -7 and -9 upregulation}

We next evaluated the role of lncZEB1-AS1 as a regulator of the epithelial-mesenchymal transition (EMT), in HCC cells, given that this transition is a key step in tumor cell oncogenic progression. No effect of lncZEB1-AS1 knockdown on the expression of the EMT-associated genes vimentin, N-cadherin, or E-cadherin was observed (Supplemental Figure 1A and B), suggesting that this lncRNA does not directly impact EMT progression. We therefore next assessed the relationship between lncZEB1-AS1 and the expression of matrix metalloproteinases (MMPs), given that they are essential mediators of tumordriven proteolysis of the extracellular matrix and subsequent invasion. We found that knockdown of lncZEB1-AS1 was associated with a significant reduction in the mRNA level expression MMP2, MMP7, and MMP9 (Figure 3A and B), and with a marked decrease in AKT phosphorylation (Figure 3C) in both tested HCC cell lines. When we ectopically expressed pmyr-AKT in cells in which lncZEB1-AS1 had been knocked down, this was associated with significant upregulation of MMP2, MMP7, and MMP9 at the mRNA level (Figure 3D). When we additionally conducted kinase activity assays and Western blotting, we were able to confirm that PI3K activity was regulated by lncZEB1-AS1, whereas this lncRNA had no comparable effect on phosphatase and tensin homolog deleted on chromosome 10 (PTEN) (Figure $3 \mathrm{E}$ and $\mathrm{F})$. These results thus suggest that lncZEB1AS1 functions as a promoter of HCC metastasis at least in part through a PI3K-AKT-dependent mechanism associated with the enhanced upregulation of proteolytic MMP enzymes.

\section{LncZEB 1-AS1 targets miR-302b in order to upregulate EGFR and thereby activate PI3K-AKT signaling}

PI3K-AKT signaling pathway activation is primarily induced as a result of signaling through specific receptor tyrosine kinases (RTKs) in response to specific ligand binding events. In order to evaluate the role of RTKs in our experimental system, we treated our HCC cell lines with either hepatocyte growth factor (HGF) or EGF. We found that the knockdown of lncZEB1-AS1 markedly impaired EGF-induced AKT activation in both Huh7 and MHCC-97H cells, whereas no such changes were observed in response to HGF stimulation (Figure 4A-B). In a GEPIA analysis, we additionally determined that there was a significant positive correlation between the expression of EGFR and lncZEB-AS1 in HCC patient tumor samples (Figure $4 C)$, suggesting that this lncRNA may promote HCC progression via an EGFR-PI3K-AKT axis. In many cases, lncRNAs have been shown to act as competing endogenous RNAs (ceRNAs) capable of binding to specific miRNAs and thereby interfering with their ability to suppress target gene expression [17]. Using starBase 3.0, we were able to identify miR-302b as a putative binding target for lncZEB1-AS based on sequence complementarity (Figure 4D). Given that this miRNA has also been found to function as a tumor suppressor in the context of HCC progression through its ability to target EGFR [27], we selected it for further validation. Using a luciferase reporter assay system, we were able to confirm that a WT but not a MUT version of lncZEB1-AS1 was able to bind to agomiR-302b such that this miRNA only altered luciferase activity when transfected into cells that had been co-transfected with a WT-lncZEB1-AS1 reporter construct (Figure 4E-F). We were further able to confirm a direct interaction between lncZEB1-AS1 and miR-302b via a RIP assay which confirmed the significant enrichment of both of these RNAs in Ago2-containing immunoprecipitates but not in precipitates prepared using control IgG (Figure 4G). When lncZEB1-AS1 was knocked down in Huh7 and MHCC-97H cells, this was associated with a significant increase in miR-302b expression in these same cells (Figure 4H). These results therefore supported a model wherein lncZEB1-AS1 functions as a ceRNA for miR-302b in HCC cells. We next assessed whether lncZEB1-AS1 was able to enhance EGFR expression in HCC cells via miR-302b sequestration by knocking down this lncRNA in cells that had been transduced with either antagomiR-302b or a control antagomir construct. We initially confirmed that antagomiR-302b transfection significantly reduced miR-302b levels in both Huh7 and MHCC-97H cells (Figure 4I), after which we confirmed that antagomiR-302b transfection was sufficient to reverse mRNA (Figure 4J) and protein (Figure 4K) levels decreased in EGFR expression in cells in which 
lncZEB1-AS1 had been knocked down. Together, these findings thus indicated that lncZEB1 functions as a positive regulator of EGFR expression in HCC cells at least in part owing to its ability to function as a "molecular sponge" for miR-302b.

\section{Discussion}

Advanced solid tumors including those of the lung, breast, and prostate often metastasize to the bone [28, 29], with BM being associated with an extremely poor patient prognosis [30]. It is vital that the molecular mechanisms governing $\mathrm{HCC} \mathrm{BM}$ be better studied, as at present they are not well understood. In previous studies, elevated lncZEB1-AS1 expression has been detected in HCC tumor samples relative to ANTs, and this lncRNA has been shown to be correlated to tumor growth and metastasis in HCC patients $[18,24]$. The specific role of lncZEB1-AS in HCC BM and any underlying molecular mechanisms, however, have not been previously assessed. In this study, we therefore examined the role of lncZEB1-AS1 in HCC development and metastatic progression.
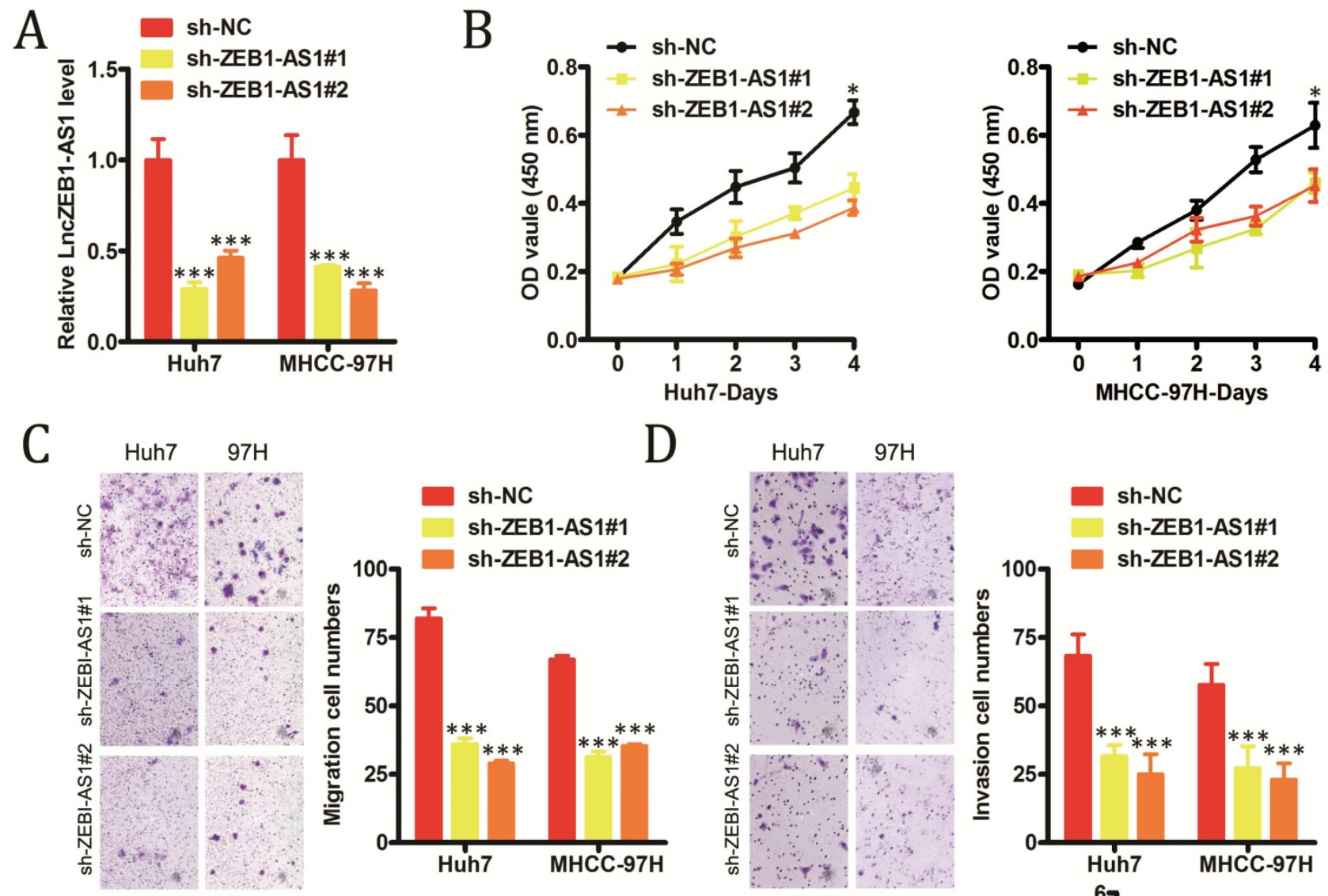

E
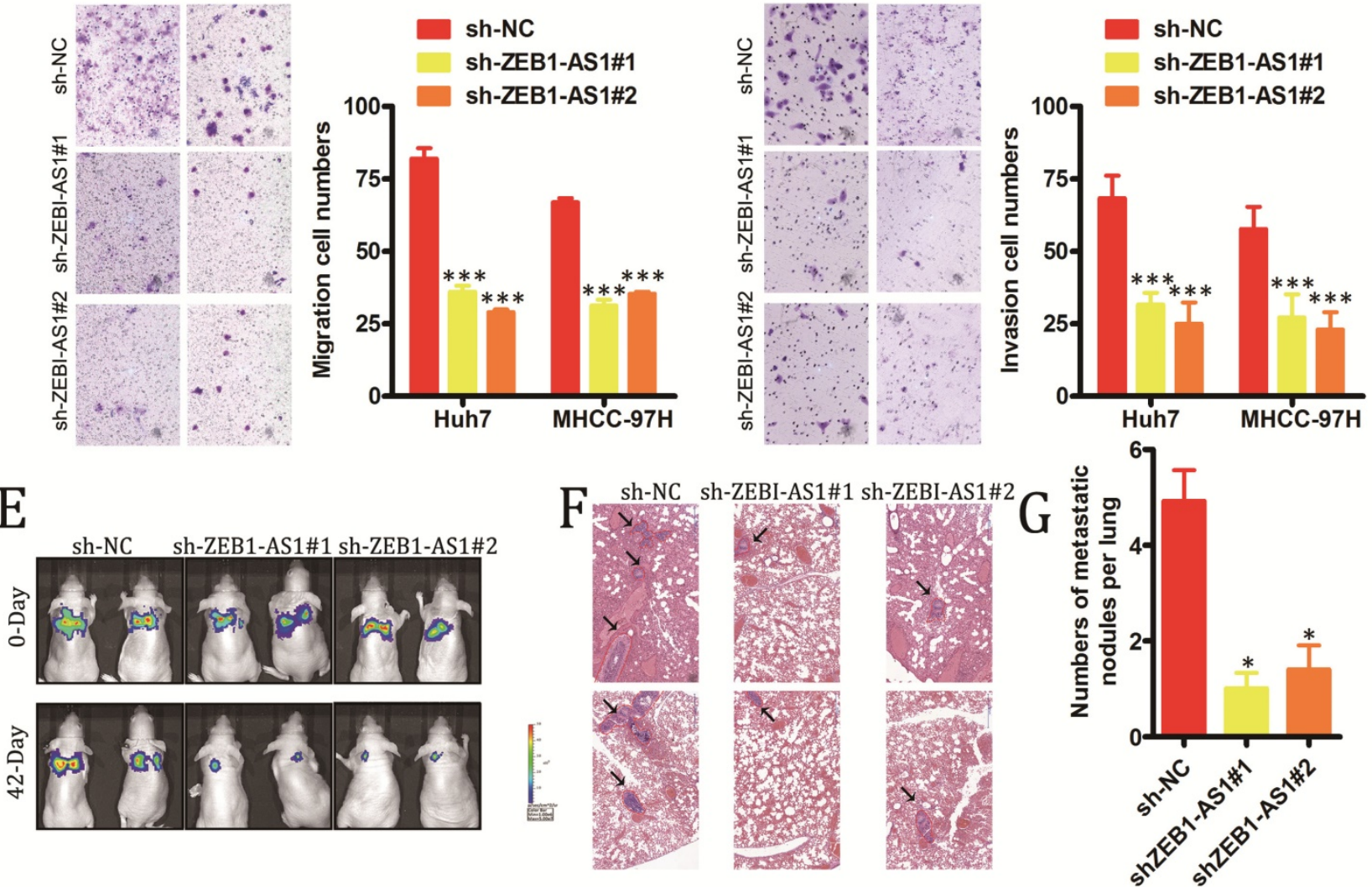

Figure 2. LncZEB1-AS1 promotes HCC cell growth and metastasis. (A) Confirmation of successful IncZEB1-AS1 knockdown (KD, shLncZEB1-AS1\#1, 2) in Huh-7 and MHCC-97H cells. (B-D) The impact of IncZEB1-AS1 knockdown on HCC cell proliferation (B). migration (C) and invasion (D). (E) Lung metastases in mice intravenously injected with HCC cells were visualized using an IVIS Imaging System on days 0 and 48 , with quantified data shown in (F). (G) Representative H\&E-stained lung metastases, with quantification shown in $(\mathbf{H})$. Scale bar, $50 \mathrm{~mm}$. Data are means \pm SEM, and were analyzed via Student's t-tests or repeated measures ANOVAs as appropriate. *p < 0.05 ; **p < $0.01 ; * * * p<0.001$. 

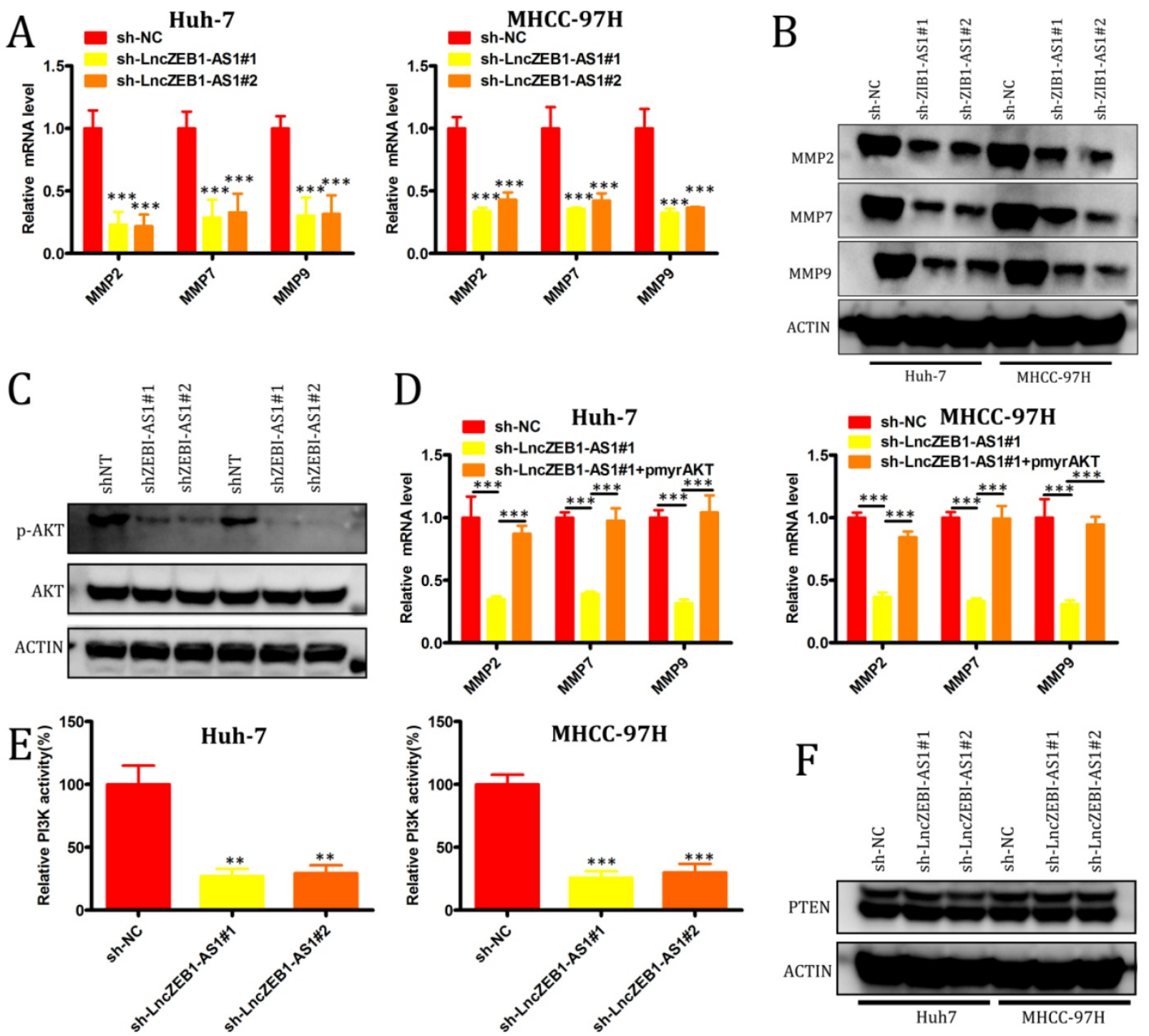

Figure 3. LncZEB I-ASI controls MMP-2, -7, and -9 expression via the activation of PI3K-AKT signaling. (A and B) qRT-PCR and Western blotting were used to quantify MMP-2, -7, and -9 levels in HCC cells. (C) Total AKT expression and AKT phosphorylation (p-AKT) were assessed in the indicated cells via Western blotting. (D) Relative MMP-2, MMP-7, and MMP-9 expression in cells treated as above was assessed via QRT-PCR. (E) PI3K activity was assessed in the indicated cells. (F) PTEN expression was assessed by Western blotting in the indicated cells. GAPDH expression was used for normalization in all experiments. Data are means \pm SEM, and were compared via Student's t-tests. ${ }^{*} \mathrm{p}<0.05 ;{ }^{* *} \mathrm{p}<0.01 ;{ }^{* * *} \mathrm{p}<0.001$

Initially, we confirmed that lncZEB1-AS1 was upregulated in HCC tumor tissue samples relative to healthy adjacent tissue, and we confirmed that there was a positive correlation between the expression of this lncRNA and the incidence of BM in HCC patients. We next examined the molecular mechanisms whereby lncZEB1-AS1 impacts HCC metastatic progression. While EMT is a key process related to tumor cell metastasis [31-34], we found that knocking down lncZEB1-AS1 did not alter EMT-related protein expression in HCC cell lines. However, we did find that changes in lncZEB1-AS1 expression were closely linked to changes in AKT activation and associated signaling in these cells. Through kinase activity assays and qRT-PCR, we ultimately found that lncZEB1-AS1 can enhance PI3K-AKT signaling in order to upregulate MMP2, MMP7, and MMP9 in HCC cells, thereby promoting tumor metastasis, consistent with prior reports highlighting the importance of AKT signaling in HCC onset and progression [35-41]. Our data further suggested that lncZEB1-AS1 promotes EGFR upregulation in HC cells, thereby enhanced EGF- but not HGF-mediated activation of PI3K-AKT signaling. While lncRNAs can alter tumor progression through several different mechanisms, the best understood such mechanism is that in which lncRNAS serve as ceRNAs for specific target miRNAs [42]. In prior studies, lncZEB1-AS1 has been shown to serve as a ceRNA for miR-133b [43], miR-942 [44], miR-455-3p [45], miR-141-3p [30], miR-409-3p [46], miR-1224-5p [47], and miR-181a-5p [48] in a range of tumor types. Through predictive analyses, we identified miR-302b as a novel lncZEB1-AS1 target miRNA that has also previously been shown to 
function as a tumor suppressor in HCC owing to its ability to target EFGR [27]. Through luciferase reporter and RIP assays we were able to confirm that lncZEB1-AS1 can directly interact with miR-302b in HCC cells, and we were additionally able to confirm that lncZEB1-AS1 knockdown significantly increased miR-302b expression in these HCC cells, thereby resulting in EGFR downregulation. Together, our results therefore support a model in which lncZEB1AS1 functions as a ceRNA for miR-302b in order to indirectly regulate EGFR expression in HCC cells.

EGFR signaling is known to be associated with many different solid tumor types including colon cancer, non-small cell lung cancer (NSCLC), breast cancer, renal cancer, head and neck cancer, and glioma [49-51]. In NSCLC, for example, EGFR overexpression is correlated with patient histopathological findings, and with the degree of tumor aggression and invasion. As a result, many studies have sought to interfere with EGFR signaling pathways in tumors in which this signaling pathway is dysregulated, resulting in beneficial clinical outcomes in multiple different solid tumor types [52-54]. Although further research is needed in order to extend these findings to $\mathrm{HCC}$, there is increasingly robust preclinical evidence suggesting that EGFR-targeting agents may be effective in the treatment or prevention of HCC $[55,56]$. Our findings further suggest that compounds targeting the lncZEB1-AS1-miR-302b-EGFR axis may be valuable as a means of preventing BM in HCC patients.
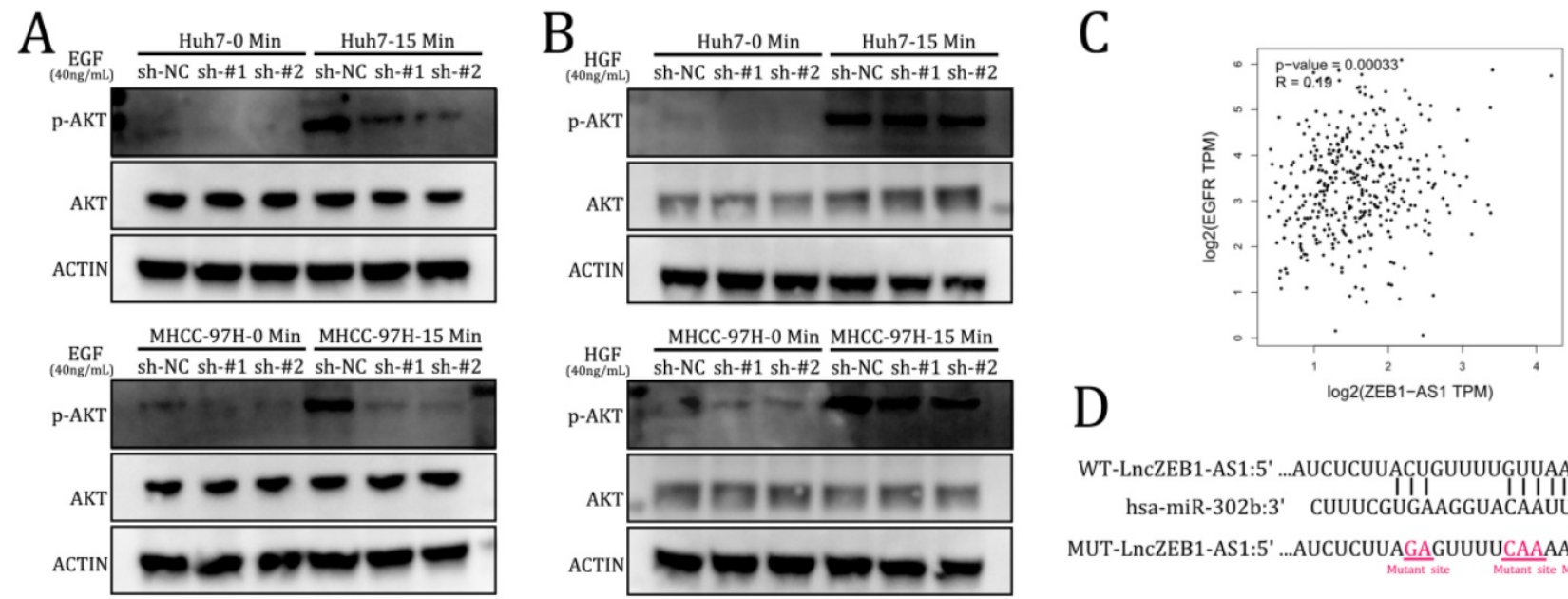

D

WT-LnCZEB1-AS1:5' ...AUCUCUUACUGUUUUGUUAAAGA. hsa-miR-302b:3' CUUUCGUGAAGGUACÁAUUUCA

MUT-LnCZEB1-AS1:5' ...AUCUCUUAGAGUUUUCAAAAACA..
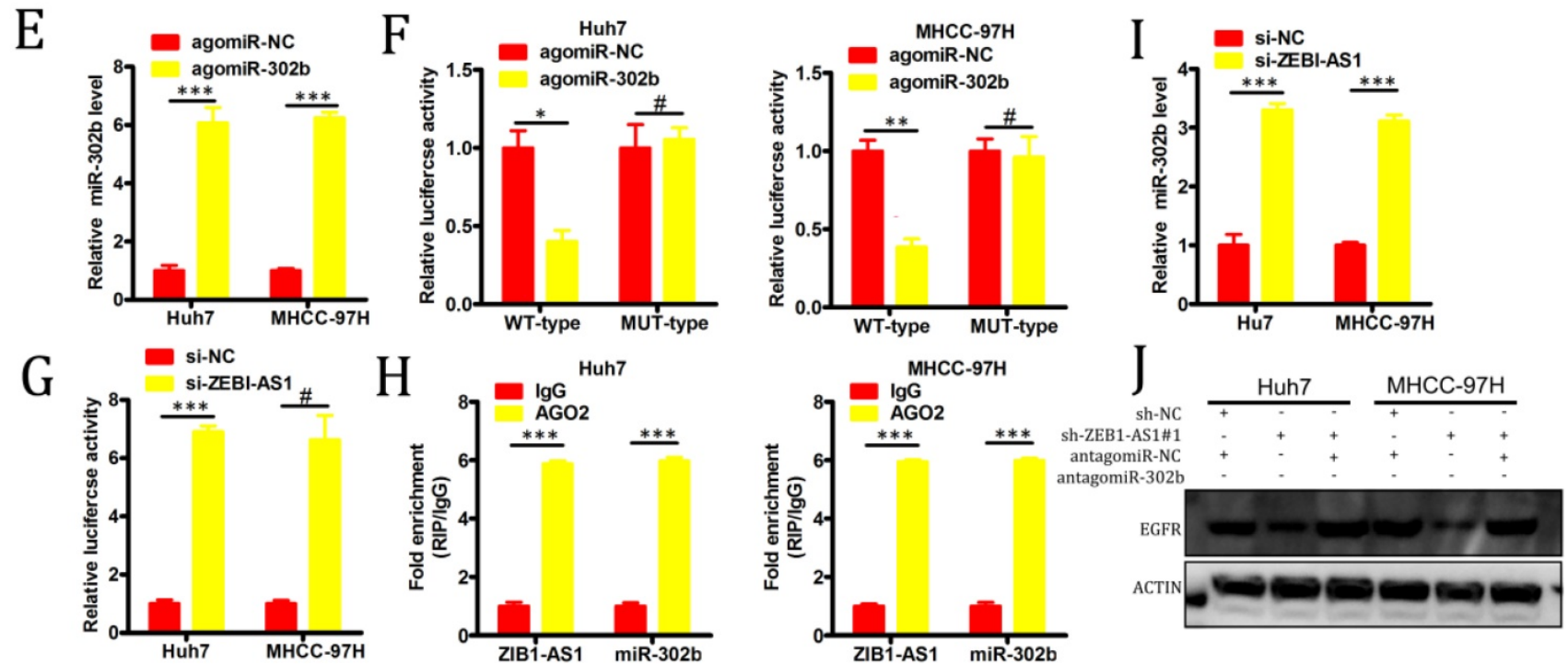

Figure 4. LncZEB1-AS1 targets miR-302b in order to upregulate EGFR and to thereby promote PI3K-AKT signaling. (A and B) Cells were treated using EGF (A) or HGF (B) for 0 - 15 minutes, after which Western blotting was conducted in order to quantify the expression of the indicated proteins. GAPDH served as a loading control. (C) A positive correlation between EGFR and IncZEBI-ASI expression was detected in the GEPIA dataset via Pearson's correlation test. (D) Bioinformatic analyses predicted an interaction between miRNA-302b and IncZEB1-AS1. (E) The expression of miRNA-302b in Huh7 and MHCC-97H cells was assessed following agomir-302b or agomir-NC transfection. (F) Wild type (WT)-IncZEB1-AS1 or mutant (MUT)-IncZEB1-AS1 were co-transfected into HCC cells together with agomir-302b p or agomir-NC. After 48 h, luciferase activity was assessed. (G) Increased ZEB1-ASI and miR-302b levels were evident in Ago2-containing immunoprecipitates relative to precipitates prepared using a control IgG. (I) qRT-PCR was used to assess miR-302b expression in HCC cells following antagomir-302b or antagomir-NC transfection. (J, K) EGFR expression was assessed via qRT-PCR and Western blotting following the co-transfection of HCC cells with si-ZEB1-AS and antagomir-302b or antagomir-NC. $* \mathrm{P}<0.05$; $* * \mathrm{P}<0.01$; $* * * \mathrm{P}<0.001$; $\# \mathrm{P}>0.05$ 
In summary, we have provided novel evidence suggesting that lncZEB1-AS1 is an important regulator of HCC BM. We have additionally highlighted a previously undescribed lncZEB1-AS1miR-302b-EGFR-PI3K-AKT signaling axis that may govern this metastatic process. This axis may be a viable target for therapeutic intervention in HCC patients as a means of significantly improving quality of life and extending patient survival.

\section{Supplementary Material}

Supplementary figure S1.

http://www.jcancer.org/v11p5118s1.pdf

\section{Acknowledgements}

\section{Funding}

The study was funded by the Project of Shanghai Science and Technology Commission (7411960100).

\section{Competing Interests}

The authors have declared that no competing interest exists.

\section{References}

1. Llovet JM, Zucman-Rossi J, Pikarsky E, Sangro B, Schwartz M, Sherman M, et al. Hepatocellular carcinoma. Nat Rev Dis Primers. 2016; 2: 16018

2. Forner A, Reig M, Bruix J. Hepatocellular carcinoma. Lancet. 2018; 391: 1301-14.

3. Llovet JM, Villanueva A, Lachenmayer A, Finn RS. Advances in targeted therapies for hepatocellular carcinoma in the genomic era. Nat Rev Clin Oncol. 2015; 12 : 408-24.

4. Longo V, Brunetti O, D'Oronzo S, Ostuni C, Gatti P, Silvestris F. Bone metastases in hepatocellular carcinoma: an emerging issue. Cancer Metastasis Rev. 2014; 33: 333-42.

5. Natsuizaka M, Omura T, Akaike T, Kuwata Y, Yamazaki K, Sato T, et al. Clinical features of hepatocellular carcinoma with extrahepatic metastases. J Gastroenterol Hepatol. 2005; 20: 1781-7.

6. Aino H, Sumie S, Niizeki T, Kuromatsu R, Tajiri N, Nakano M, et al. Clinical characteristics and prognostic factors for advanced hepatocellular carcinoma with extrahepatic metastasis. Mol Clin Oncol. 2014; 2: 393-8.

7. Xiang ZL, Zeng ZC, Tang ZY, Fan J, He J, Zeng HY, et al. Potential prognostic biomarkers for bone metastasis from hepatocellular carcinoma. Oncologist. 2011; 16: 1028-39.

8. Roodman GD. Mechanisms of bone metastasis. N Engl J Med. 2004; 350: 1655-64.

9. He J, Zeng ZC, Tang ZY, Fan J, Zhou J, Zeng MS, et al. Clinical features and prognostic factors in patients with bone metastases from hepatocellular carcinoma receiving external beam radiotherapy. Cancer. 2009; 115: 2710-20.

10. Ponting $\mathrm{CP}$, Oliver PL, Reik W. Evolution and functions of long noncoding RNAs. Cell. 2009; 136: 629-41.

11. Pandey GK, Mitra S, Subhash S, Hertwig F, Kanduri M, Mishra K, et al. The risk-associated long noncoding RNA NBAT-1 controls neuroblastoma progression by regulating cell proliferation and neuronal differentiation. Cancer Cell. 2014; 26: 722-37.

12. Michalik KM, You X, Manavski Y, Doddaballapur A, Zornig M, Braun T, et al. Long noncoding RNA MALAT1 regulates endothelial cell function and vessel growth. Circ Res. 2014; 114: 1389-97.

13. Li L, Liu B, Wapinski OL, Tsai MC, Qu K, Zhang J, et al. Targeted disruption of Hotair leads to homeotic transformation and gene derepression. Cell Rep. 2013; 5: 3-12.

14. Liu M, Sun W, Liu Y, Dong X. The role of IncRNA MALAT1 in bone metastasis in patients with non-small cell lung cancer. Oncol Rep. 2016; 36: 1679-85.

15. Li C, Wang S, Xing Z, Lin A, Liang $\mathrm{K}$, Song J, et al. A ROR1-HER3-lncRNA signalling axis modulates the Hippo-YAP pathway to regulate bone metastasis. Nat Cell Biol. 2017; 19: 106-19.

16. Derrien T, Johnson R, Bussotti G, Tanzer A, Djebali S, Tilgner H, et al. The GENCODE v7 catalog of human long noncoding RNAs: Analysis of their gene structure, evolution, and expression. Genome Res. 2012; 22: 1775-89.

17. Mercer TR, Dinger ME, Mattick JS. Long non-coding RNAs: insights into functions. Nature Reviews Genetics. 2009; 10: 155-9.
18. Li T, Xie J, Shen C, Cheng D, Shi Y, Wu Z, et al. Upregulation of long noncoding RNA ZEB1-AS1 promotes tumor metastasis and predicts poor prognosis in hepatocellular carcinoma. Oncogene. 2016; 35: 1575-84.

19. Li JL, Li ZL, Leng KM, Xu Y, Ji DL, Huang LN, et al. ZEB1-AS1: A crucial cancer-related long non-coding RNA. Cell Prolif. 2018; 51: e12423.

20. Wang YL, Bai Y, Yao WJ, Guo L, Wang ZM. Expression of long non-coding RNA ZEB1-AS1 in esophageal squamous cell carcinoma and its correlation with tumor progression and patient survival. Int J Clin Exp Patho. 2015; 8: 11871-6.

21. Xiong WC, Han N, Wu N, Zhao KL, Han C, Wang HX, et al. Interplay between long noncoding RNA ZEB1-AS1 and miR-101/ZEB1 axis regulates proliferation and migration of colorectal cancer cells. Am J Transl Res. 2018; 10: 605-17.

22. Pastushenko I, Blanpain C. EMT Transition States during Tumor Progression and Metastasis. Trends Cell Biol. 2019; 29: 212-26.

23. Li YL, Wen XW, Wang LG, Sun XJ, Ma H, Fu Z, et al. LncRNA ZEB1-AS1 predicts unfavorable prognosis in gastric cancer. Surg Oncol. 2017; 26: 527-34.

24. Li MF, Guan H, Liu YP, Gan XF. LncRNA ZEB1-AS1 reduces liver cancer cell proliferation by targeting miR-365a-3p. Exp Ther Med. 2019; 17: 3539-47.

25. Zheng $H$, Yang $Y$, Hong YG, Wang MC, Yuan SX, Wang ZG, et al. Tropomodulin 3 modulates EGFR-PI3K-AKT signaling to drive hepatocellular carcinoma metastasis. Mol Carcinogen. 2019; 58: 1897-907.

26. Ye QH, Zhu WW, Zhang JB, Qin Y, Lu M, Lin GL, et al. GOLM1 Modulates EGFR/RTK Cell-Surface Recycling to Drive Hepatocellular Carcinoma Metastasis. Cancer Cell. 2016; 30: 444-58.

27. Wang LM, Yao JY, Shi X, Hu LL, Li ZF, Song TS, et al. MicroRNA-302b suppresses cell proliferation by targeting EGFR in human hepatocellular carcinoma SMMC-7721 cells. BMC Cancer. 2013; 13: 448.

28. Huang W, Su X, Yan W, Kong Z, Wang D, Huang Y, et al. Overexpression of AR-regulated lncRNA TMPO-AS1 correlates with tumor progression and poor prognosis in prostate cancer. Prostate. 2018; 78: 1248-61.

29. Wang W, Lou W, Ding B, Yang B, Lu H, Kong Q, et al. A novel mRNA-miRNA-lncRNA competing endogenous RNA triple sub-network associated with prognosis of pancreatic cancer. Aging (Albany NY). 2019; 11: 2610-27.

30. Qian W, Cai X, Qian Q, Peng W, Yu J, Zhang X, et al. IncRNA ZEB1-AS1 promotes pulmonary fibrosis through ZEB1-mediated epithelial-mesenchymal transition by competitively binding miR-141-3p. Cell Death Dis. 2019; 10: 129.

31. Brabletz T. EMT and MET in Metastasis: Where Are the Cancer Stem Cells? Cancer Cell. 2012; 22: 699-701.

32. Zheng HQ, Shen MH, Zha YL, Li WY, Wei Y, Blanco MA, et al. PKD1 Phosphorylation-Dependent Degradation of SNAIL by SCF-FBXO11 Regulates Epithelial-Mesenchymal Transition and Metastasis. Cancer Cell. 2014; 26: 358-73.

33. Mak P, Leav I, Pursell B, Bae D, Yang X, Taglienti CA, et al. ERbeta impedes prostate cancer EMT by destabilizing HIF-1alpha and inhibiting VEGF-mediated snail nuclear localization: implications for Gleason grading. Cancer Cell. 2010; 17: 319-32.

34. Singh A, Greninger P, Rhodes D, Koopman L, Violette S, Bardeesy N, et al. A Gene Expression Signature Associated with "K-Ras Addiction" Reveals Regulators of EMT and Tumor Cell Survival. Cancer Cell. 2009; 15: 489-500.

35. Zuo XL, Chen ZQ, Cai J, Gao W, Zhang Y, Han GY, et al. 5-Hydroxytryptamine Receptor 1D Aggravates Hepatocellular Carcinoma Progression Through FoxO6 in AKT-Dependent and Independent Manners. Hepatology. 2019; 69: 2031-47.

36. Wang S, Wang JQ, Lv XW. Exosomal miRNAs as biomarkers in the diagnosis of liver disease. Biomark Med. 2017; 11: 491-501.

37. Wang SS, Zhu MY, Wang QY, Hou YL, Li L, Weng HL, et al. Alpha-fetoprotein inhibits autophagy to promote malignant behaviour in hepatocellular carcinoma cells by activating PI3K/AKT/mTOR signalling. Cell Death Dis. 2018; 9: 1027.

38. Wu Q, Chen JX, Chen Y, Cai LL, Wang XZ, Guo WH, et al. The chemokine receptor CCR10 promotes inflammation-driven hepatocarcinogenesis via PI3K/Akt pathway activation. Cell Death Dis. 2018; 9: 232.

39. Chaturantabut S, Shwartz A, Evason KJ, Cox AG, Labella K, Schepers AG, et al. Estrogen Activation of G-Protein-Coupled Estrogen Receptor 1 Regulates Phosphoinositide 3-Kinase and mTOR Signaling to Promote Liver Growth in Zebrafish and Proliferation of Human Hepatocytes. Gastroenterology. 2019;156:1788-1804.e13.

40. Klingenberg M, Gross M, Goyal A, Polycarpou-Schwarz M, Miersch T, Ernst AS, et al. The Long Noncoding RNA Cancer Susceptibility 9 and RNA Binding Protein Heterogeneous Nuclear Ribonucleoprotein L Form a Complex and Coregulate Genes Linked to AKT Signaling. Hepatology. 2018; 68: 1817-32.

41. Yang N, Zhou J, Li Q, Han F, Yu Z. miR-96 exerts carcinogenic effect by activating AKT/GSK-3beta/beta-catenin signaling pathway through targeting inhibition of FOXO1 in hepatocellular carcinoma. Cancer Cell Int. 2019; 19: 38.

42. Paraskevopoulou MD, Hatzigeorgiou AG. Analyzing MiRNA-LncRNA Interactions. Methods Mol Biol. 2016; 1402: 271-86.

43. Jiang X, Li J, Wang W, Hu Z, Guan C, Zhao Y, et al. AR-induced ZEB1-AS1 represents poor prognosis in cholangiocarcinoma and facilitates tumor stemness, proliferation and invasion through mediating miR-133b/HOXB8. Aging (Albany NY). 2020; 12: 1237-55.

44. Hua Z, Ma K, Liu S, Yue Y, Cao H, Li Z. LncRNA ZEB1-AS1 facilitates ox-LDL-induced damage of HCtAEC cells and the oxidative stress and 
inflammatory events of THP-1 cells via miR-942/HMGB1 signaling. Life Sci. 2020;247:117334.

45. Ni X, Ding Y, Yuan H, Shao J, Yan Y, Guo R, et al. Long non-coding RNA ZEB1-AS1 promotes colon adenocarcinoma malignant progression via miR-455-3p/PAK2 axis. Cell Prolif. 2020; 53: e12723.

46. Qu RZ, Chen XM, Zhang C. LncRNA ZEB1-AS1/miR-409-3p/ZEB1 feedback loop is involved in the progression of non-small cell lung cancer. Biochem Bioph Res Co. 2018; 507: 450-6.

47. Wang $\mathrm{Q}$, Zhang RR, Liu DD. Long non-coding RNA ZEB1-AS1 indicates poor prognosis and promotes melanoma progression through targeting miR-1224-5p. Exp Ther Med. 2019; 17: 857-62.

48. Lv SY, Shan TD, Pan XT, Tian ZB, Liu XS, Liu FG, et al. The IncRNA ZEB1-AS1 sponges miR-181a-5p to promote colorectal cancer cell proliferation by regulating Wnt/beta-catenin signaling. Cell Cycle. 2018; 17: 1245-54.

49. Fujino S, Enokibori T, Tezuka N, Asada Y, Inoue S, Kato H, et al. A comparison of epidermal growth factor receptor levels and other prognostic parameters in non-small cell lung cancer. Eur J Cancer. 1996; 32A: 2070-4.

50. Herbst RS, Langer CJ. Epidermal growth factor receptors as a target for cancer treatment: The emerging role of IMC-C225 in the treatment of lung and head and neck cancers. Semin Oncol. 2002; 29: 27-36.

51. Kondapaka SB, Fridman R, Reddy KB. Epidermal growth factor and amphiregulin up-regulate matrix metalloproteinase-9 (MMP-9) in human breast cancer cells. Int J Cancer. 1997; 70: 722-6.

52. Giaccone G. Targeting HER1/EGFR in cancer therapy: experience with erlotinib. Future Oncol. 2005; 1: 449-60.

53. Ethier SP. Signal transduction pathways: the molecular basis for targeted therapies. Semin Radiat Oncol. 2002; 12: 3-10.

54. Baselga J, Arteaga CL. Critical update and emerging trends in epidermal growth factor receptor targeting in cancer. J Clin Oncol. 2005; 23: 2445-59.

55. Schiffer E, Housset C, Cacheux W, Wendum D, Desbois-Mouthon C, Rey C, et al. Gefitinib, an EGFR inhibitor, prevents hepatocellular carcinoma development in the rat liver with cirrhosis. Hepatology. 2005; 41: 307-14.

56. Berasain C, Castillo J, Prieto J, Avila MA. New molecular targets for hepatocellular carcinoma: the ErbB1 signaling system. Liver Int. 2007; 27: $174-85$ 